\title{
Study on Bottlenecks in Airport Security Process Based on Simulation
}

\author{
Yaxin Liu \\ North China Electric Power University, Baoding, Hebei Province, China \\ 18730266367@163.com
}

\begin{abstract}
Based on the advanced experience at home and abroad, this paper discusses the bottleneck identification method of airport security inspection process with airport security process as the research object. Firstly, we use MATLAB to establish a simulation model and introduce the number of passenger, the security line service rate and the region's largest stranded number. And based on TOC theory, we establish the airport simulation queuing system model. Then we put all the parameters into the simulation model to get three function models. After that, we load a certain number of passengers to get three function values of each line. Then we run the model and combined with the data, we can determine the location of the bottleneck. At the end, we reach a decision that the main bottleneck in the current security process is in the mm wave process.
\end{abstract}

Key words: MATLAB; TOC theory; simulation queuing system model; airport security process; bottleneck; mm.

\section{INTRODUCTION}

With the increasing number of people traveling, the plane is chosen by more and more people and security problems in the process gradually exposed, which mainly include that screening process takes too long time and the passengers waste a lot of time in the process of security, so people take more and more attention to this phenomenon and hope it to be solved.so a dynamic bottleneck method is proposed in this paper to establish the dynamic bottleneck of the security process by establishing a simulation model. The dynamic bottleneck identification method is based on the simulation platform to obtain the required data and can identify various types of bottlenecks in the security process and make clear the dependencies and importance among these bottlenecks.

\section{ESTABLISHMENT OF SIMULATION MODEL}

\section{Data Preparation and Acquisition.}

Firstly, we should identify the bottleneck object, the bottleneck of this paper identifies the airport security throughput of different time periods (peak, flat peak) under different passenger flow conditions (normal passenger flow, large passenger flow). Then, we build the airport security bottleneck identification index set. A hierarchical index set for dynamic bottleneck identification of airport security is constructed, and the meaning of each index and its calculation formula are indicated. As shown below:

Then, we select the simulation tool and build the security simulation model. Aiming at the established dynamic bottleneck identification object and the corresponding bottleneck identification index set, the simulation tool MATLAB needed to realize the bottleneck identification is determined, and the corresponding airport security simulation model is established by MATLAB. And we design the simulation scheme, including the airport security conditions of the passenger station, staffing, opening number, equipment and facilities operation and so on. The required data are shown in Table 2. 
TABLE 1. Airport security bottleneck identification of dynamic bottlenecks

\begin{tabular}{|c|c|c|c|c|}
\hline criterion & $\begin{array}{l}\text { Bottleneck identification } \\
\text { indicators }\end{array}$ & Index purpose & $\begin{array}{l}\text { Computational } \\
\text { formula }\end{array}$ & Target object \\
\hline \multirow{2}{*}{ efficiency } & $\begin{array}{l}\text { the overall system } \\
\text { efficiency } E_{S}\end{array}$ & $\begin{array}{l}\text { the whole system } \\
\text { security number per } \\
\text { unit time }\end{array}$ & $E_{S}$ & entirety \\
\hline & $\begin{array}{c}\text { efficiency of each point } \\
\qquad E_{i}\end{array}$ & $\begin{array}{l}\text { each security } \\
\text { checkpoints security } \\
\text { number per unit time }\end{array}$ & $E_{i}$ & $\begin{array}{l}\text { a separate } \\
\text { security } \\
\text { checkpoint }\end{array}$ \\
\hline \multirow{2}{*}{ smoothness } & $\begin{array}{c}\text { the region's biggest } \\
\text { passenger Numbers } D_{\max }^{i}\end{array}$ & $\begin{array}{l}\text { passengers maximum } \\
\text { moment area }\end{array}$ & $D_{\max }^{i}=\operatorname{MAX}\left\{D_{\tau}^{i}\right\}$ & $\begin{array}{l}\text { a separate } \\
\text { security } \\
\text { checkpoint }\end{array}$ \\
\hline & $\begin{array}{c}\text { regional average dead } \\
\text { time } T_{a v e}^{i}\end{array}$ & $\begin{array}{l}\text { passengers in the } \\
\text { stagnation of the } \\
\text { regional average time }\end{array}$ & $T_{a v e}^{i}=A V E\left\{T_{n w}^{i}\right\}$ & $\begin{array}{l}\text { a separate } \\
\text { security } \\
\text { checkpoint }\end{array}$ \\
\hline
\end{tabular}

TABLE 2. The required data

\begin{tabular}{|c|c|c|}
\hline \multirow{2}{*}{ Equipment and facilities } & \multicolumn{2}{|c|}{ The required data } \\
\cline { 2 - 3 } & Arrival/ By the average time(min) & $\begin{array}{c}\text { A single zone stranded maximum } \\
\text { number of passengers }\end{array}$ \\
\hline The entrance & 0.200 & $\infty$ \\
\hline ID check & 0.375 & 150 \\
\hline mm wave & 0.436 & 50 \\
\hline X-ray & 0.412 & 50 \\
\hline
\end{tabular}

After the design of the simulation program, the airport security system can be carried out passenger simulation process simulation, access to simulation results data.

\section{Identification of Potential Bottlenecks.}

We calculate the value of the bottleneck identification index and analyze the value of the index According to the simulation result data and the calculation formula of the index, the value of each bottleneck identification index is calculated, and the numerical results of each index are analyzed [3]. the meaning of each index and its calculation formula are indicated. As shown below:

Based on the average waiting time: make the longest average waiting time in the security point as the bottleneck. The mathematical expression is

$$
B=\left\{j \mid T_{\text {avej }}=\max \left(T_{\text {ave } 1} T_{\text {ave } 2,} \cdots, T_{\text {avem }}\right\}\right.
$$

Based on the average utilization rate: make the highest average utilization rate in the security point as the bottleneck. The mathematical expression is

$$
B=\left\{j \mid \rho_{j}=\max \left(\rho_{1}, \rho_{2}, \cdots, \rho_{m}\right\}\right.
$$




$$
\rho_{j}=\frac{\lambda_{j}}{v_{j}}
$$

Where:

B: Bottleneck

$\rho_{j}:$ The first $\mathrm{j}$ security checkpoints' utilization

$\lambda_{j}$ : The passengers' arrival rate of security checkpoints $\mathrm{j}$

$v_{j}$ : The service rate of security checkpoints $\mathrm{j}$

Based on Sensitivity: Find the security point that the self-change has the greatest influence on the whole system and set the security point with the biggest sensitivity value of the whole system's service rate as the bottleneck.

The overall service rate of the system $P_{S}$ is a function of the service rate of each security point $P_{j}$, The mathematical expression is

$$
P_{S}=P_{S}\left(P_{1}, P_{2}, \cdots, P_{m}\right)
$$

At this point, the bottleneck of the system is

$$
B=\left\{i \| \frac{\partial P_{S}}{P_{j}}|>| \frac{\partial P_{S}}{P_{i}} \mid, \forall i \neq j\right\}
$$

Then, I determine the threshold of indicators. From the security bottleneck identification of dynamic bottlenecks, select a number of evaluation indicators, the threshold value of bottleneck identification index in this state is determined according to the current simulation passenger flow condition, and the values of these indicators are compared with a given threshold, determine if this process exceeds the threshold. And we determine $n$ potential bottlenecks in the security process. According to the previous step in the security of each step-by-step flow of the target threshold to determine the results, determine $n$ potential bottlenecks in the security process, and mark these $n$ potential bottlenecks as $D_{i}(\mathrm{i}=1,2, \ldots, \mathrm{n})$

\section{Identify the main Bottleneck.}

We obtain secondary simulation result data from each potential bottleneck process. For each potential bottleneck process, increase the number of units, load the original passenger flow for the second simulation, access to the potential bottlenecks of the second simulation results data. Then, I calculate the sensitivity of the potential bottlenecks and indicators of the value of the process. Based on the data of secondary simulation results of potential bottlenecks, the sensitivity coefficients are calculated and the bottleneck identification indexes of other potential processes under the simulation results are calculated. Then, I determine the main bottleneck flow of airport security the potential bottleneck in accordance with the sensitivity of the value of the process in descending order, then the maximum value of the sensitive process is the main bottleneck process of the security.

\section{Subordinate Bottleneck of the main Bottleneck Identification.}

I extract the secondary simulation result data of the main bottleneck flow, target thresholds are determined for potential bottlenecks associated with the main bottleneck streamline, the process that no longer exceeds the threshold is the subordinate bottleneck process of the primary bottleneck. 


\section{Secondary Bottleneck and its subordinate Bottleneck Identification.}

I identify the security bottleneck process. The remaining number of potential bottlenecks in the process is calculated according to the sensitivity coefficient in descending order, then the maximum value of the sensitive process is the secondary bottleneck process of the security. Then, I classify and determine the potential bottlenecks. And I determine whether there is unclassified potential bottleneck flow at this time, if it exists, execute the previous step; If it does not exist, the next step is executed.

\section{Bottleneck Recognition Result.}

Aiming at the sub-bottleneck and secondary bottleneck identification of the main bottleneck and the main bottleneck in the process of airport security inspection, the results of dynamic bottleneck identification are given. As shown in Table 3.

TABLE 3. Security bottleneck identification results

\begin{tabular}{|c|c|c|c|}
\hline Security checkpoints & $\begin{array}{c}\text { The average passenger } \\
\text { waiting time (min) }\end{array}$ & The average utilization (\%) & The sensitivity \\
\hline The entrance & 10.3783 & 73. & 0.3357 \\
\hline ID check & 12.8212 & 79 & 0.4572 \\
\hline mm wave & 19.8472 & 88 & 0.6324 \\
\hline X-ray & 13.4351 & 82 & 0.4899 \\
\hline
\end{tabular}

\section{SUMMARY}

From the results, we can come to the conclusion that the main bottleneck in the current security process is in the $\mathrm{mm}$ wave process, X-ray is its subordinate bottleneck, while ID check is the secondary bottleneck.

\section{REFERENCES}

1. X.H. Liu, L. Zhang, Y. Zhao and Q. Zhang: Technology and Innovation, Vol. 35 (2017) No.9, p.125. (In Chinese)

2. L.Q. Lin and J. Chang: International Symposium on Assembly and Manufaturing, Vol. 1 (2007) No.9, p.130.

3. Y.C. Wang, Q.C. Zhao and D.Z. Zheng: Journal of Systems Science and Systems Engineering, Vol. 14 (2005) No.3, p.347. (In Chinese).

4. R.D. Lange, I.S. Lovich and B.D. Rhee: European Journal of Operational Research, Vol. 225 (2013) No.1, p.153.

5. R. Bian: Optimization of Passengers' Baggage Transportation for Domestic Aviation Industry (MS., Huazhong University of Science \&Technology, China 2013), p.40. (In Chinese).

6. X.X. Ning: The Course of Practical Operations Research (Beijing Science Press, China 2007). (In Chinese).

7. Z.W. Zhao and J.J. Ma: Comprehensive Transportatio, Vol. 38 (2016) No.10, p.64. (In Chinese).

8. H.Y. Li, T.S. Chen, F. Yan and L.H. Yang: Economic and Trade Practice, Vol. 351 (2017) No.11, p.14. (In Chinese).

9. M.C. Sun: Journal of Huanggang Vocational and Technical College. Vol. 354 (2017) No.5, p.122. (In Chinese)

10. Y.C. Cao, P.W. Chen, K.B. Lv, X. Gao and S.S. Cao: Mathematical Modeling and its Application, Vol. 6 (2017) No.2, p.76. (In Chinese). 